\title{
Maternal and perinatal morbidity and mortality in COVID-19 positive obstetric patients with medical disorders in tertiary care hospital in North India
}

\author{
Gunjan Goyal $^{1}$, Khushboo Tongaria ${ }^{2 *}$, Sachin Kumar ${ }^{3}$, \\ Brijesh Kumar Thakran ${ }^{4}$, Mohini Yadav 5
}

\begin{abstract}
${ }^{1}$ Department of Obstetrics and Gynaecology, World College of Medical Sciences and Research, Jhajjar, Haryana, India ${ }^{2}$ Department of Obstetrics and Gynaecology, Fortis La-Femme, Greater Kailash, New Delhi, India ${ }^{3}$ Department of Pediatrics, Bansal Hospital, New Delhi, India

${ }^{4}$ Department of Internal Medicine, World College of Medical Sciences and Research, Jhajjar, Haryana, India

${ }^{5}$ Department of Pediatrics, Aarvy Hospital, Gurugram, India
\end{abstract}

Received: 25 June 2021

Accepted: 21 July 2021

\section{*Correspondence:}

Dr. Khushboo Tongaria,

E-mail: drktong91@gmail.com

Copyright: () the author(s), publisher and licensee Medip Academy. This is an open-access article distributed under the terms of the Creative Commons Attribution Non-Commercial License, which permits unrestricted non-commercial use, distribution, and reproduction in any medium, provided the original work is properly cited.

\begin{abstract}
Background: Coronavirus disease (COVID-19) is an infectious disease caused by a newly discovered coronavirus. Most people infected with the COVID-19 virus experienced mild to moderate respiratory illness. Coronavirus is known to human kind since 1930. It was first discovered in domesticated chicken with symptoms of pulmonary infection. The aim and objective was to study clinical profile of patients presented with medical disorders in COVID-19 positive pregnant women.

Methods: Present retrospective COHORT study included 50 COVID-19 positive obstetrics patients. The study was conducted in the department of obstetrics and gynaecology, World college of medical sciences and research, Jhajjar (Haryana). Retrospective review of medical records of all pregnant patients with pre-existing medical disorders or those suspected with medical disorders on the basis of clinical and/or laboratory data from 1 April 2020 through 30 September 2020.

Results: Mean age of the women was $27.46 \pm 4$.16 years with age range of 20-35 years. Mean period of gestation was $33.26 \pm 8.35$ weeks. A total of $33(66 \%)$ women were found to be COVID-19 positive due to close contact. 29 (58\%) women were found to be asymptomatic and 17 (34\%) had various other COVID-19 symptoms. In 17 (34\%), sore throat was the commonest symptoms followed by cough, myalgia and fatigue, that is, $13(26 \%)$ each. Fever at the time of admission was present in $9(18 \%)$ women and postpartum fever was observed in $4(8 \%)$ women. Mean hospital stay was $9.24 \pm 3.25$ days. A total of $29(58 \%)$ women underwent caesarean delivery and $21(42 \%)$ had normal vaginal delivery. Mean postoperative hospital stay was 4.48 \pm 2.24 days. In 9 women, postoperative complications were seen. A total of 8 babies admitted to NICU due to various complications. Two babies expired during the study period.

Conclusions: In the present retrospective COHORT study, the majority of the pregnant women with COVID-19 infection had mild symptoms with no severe illness. Mother-to-child transmission of COVID-19 cannot be ruled out. The impacts of COVID-19 infection on patients with medical disorders during pregnancy were seen.
\end{abstract}

Keywords: Maternal, Perinatal, Morbidity, Mortality, Obstetric, Medical disorders 


\section{INTRODUCTION}

COVID-19 is an infectious disease caused by a newly discovered coronavirus and a global burden. The disease was first reported in Wuhan city, China in December 2019. It initially considered to be spread by zoonotic transmission with high rate of human to human transmission and rapidly spread to rest of the world and declared as pandemic by WHO on 11 March $2020 .{ }^{1}$

As the people are hesitant in seeking treatment for chronic diseases because of pandemic, but obstetrics never stops, as it is a powerful gift of mother nature. The physiological changes of pregnancy are well tolerated by most of women and are reversible. ${ }^{2}$ Medical problems may interfere with the physiologic adaptations of pregnancy and cause poor pregnancy outcome and vice versa. ${ }^{3}$

Most common medical disorder was pregnancy induced hypertension and its complication seen in $43 \%$ of the females, followed by anaemia and hypothyroidism seen in $20 \%$ females respectively. ${ }^{4}$

Various type of medical disorders including cardiovascular system, respiratory system, gastrointestinal, renal, endocrine disorders, hematological disorders, central nervous system may exist before pregnancy or may present for first time during pregnancy such as pregnancy induced hypertension and gestational hypertension. The main outcome of pregnancy depends upon the nature of the disease or severity of the disease at the onset of pregnancy and the quality of obstetric and medical management used. Therefore, management of pregnancies with pre-existing medical disorders should begin before conception.

The management of medical disorders in pregnancy based on four important clinical principles. They are medical disorders affected by pregnancy; medical disorders that affect pregnancy; physiological changes may make diagnose of medical disease difficult and treatment of medical diseases may be totally different in pregnant state and non-pregnant state.

Keeping in view the above facts, the present retrospective COHORT study was planned to assess the maternal and perinatal morbidity and mortality in COVID-19 positive obstetric patients with medical disorders. The purpose of this study was to know the consequences of COVID-19 on different medical disorders in pregnancy and their effect on maternal health and foetal well-being and pregnancy affecting medical disorders and their sequel. Further the present retrospective COHORT study determined the clinical course, onset of symptoms to detection of infection, management and outcome of COVID-19 positive obstetric with medical disorders in dedicated COVID-19 hospital. Study tried to understand the interaction of COVID-19 infection with other comorbidity of pregnancy and other life threatening conditions in obstetrics and to track every high risk pregnancy and outcome of COVID-19 affected high risk patients and problems faced and their on-ground solutions.

\section{METHODS}

The present retrospective COHORT study was conducted in the department of obstetrics and gynecology, World college of medical sciences and research, Jhajjar (Haryana). Study reviewed the medical records of all pregnant patients who were admitted to obstetrics and gynaecology department with pre-existing medical disorders or those suspected with medical disorders on the basis of clinical and/or laboratory data from 1 April 2020 through 30 September 2020. All pregnant women admitted with medical disorders from time of diagnosis of COVID19 positive pregnancy till delivery was included. A total of 50 women were included over a period of 6 months.

COVID-19 was diagnosed by using standard ICMR guidelines for diagnosis and treatment of COVID-19. All COVID-19 patients were diagnosed positive by using reverse transcription polymerase chain reaction results (RT-PCR) from respiratory samples as per the institutional protocol. All required initial investigations were carried out for diagnosis and progress of medical disorders complicated by COVID-19 positive infection.

\section{Methodology}

All demographic parameters like chief complaints, past medical and obstetric history of patients were noted in detail. General and systemic examination findings were done and necessary investigations depending upon suspected underlying medical conditions were gathered. Various baseline investigations like complete blood count, peripheral blood smear, liver function test, renal function test, random blood sugar, coagulation profile, viral marker, routine urine and obstetric USG was thoroughly studied in all patients.

Patients were categorized in to three groups by standard MOH guidelines. ${ }^{5}$

\section{Group A}

Asymptomatic/patients with mild symptoms with RR: <24 $/ \mathrm{m}$ and $\mathrm{SpO}_{2}$ : $>94 \%$ in room air.

\section{Group B}

Symptomatic patients with mild to moderate pneumonia with no signs of severe disease with RR: 24-30 /m (or) $\mathrm{SpO}_{2}$ : $90-94 \%$ at room air.

\section{Group C}

Symptomatic patients with severe pneumonia with RR: $>30 /$ min (or) $\mathrm{SpO}_{2}$ : $<90 \%$ at room air (or) less than $94 \%$ with oxygen, ARDS, septic shock. 
Various medical disorders were classified system wise and categorised (Table 1).

All patients received standard medical line of management as per department protocols. Hospitalisation duration and pregnancy outcome noted for those were delivered in our institution. All patients who turned negative for COVID19 and delivered outside in non-COVID-19 institution, data was collected for same retrospectively.
WHO definitions were used to define patients with anaemia, premature birth, still birth, abortion, primigravida, multipara, maternal mortality and low birth weight. Viral RNA detection using RT-PCR is the standard for the diagnosis. Swab from saliva, nasopharynx, oropharynx, sputum, endotracheal aspirate, bronchoalveolar lavage, urine and stool sample were taken. ${ }^{6}$ As per ICMR, criteria for doing laboratory test are the same for everyone. ${ }^{7,8}$

Table 1: Various medical disorders were classified system wise and categorised.

\begin{tabular}{|c|c|}
\hline Systems & Diseases \\
\hline \multirow{4}{*}{ Cardiovascular system } & Rheumatic heart diseases \\
\hline & Pregnancy induced hypertension \\
\hline & Congenital heart diseases \\
\hline & Dilated cardiomyopathy \\
\hline \multirow{3}{*}{ Respiratory system } & Asthma \\
\hline & Acute pharyngitis \\
\hline & Pulmonary tuberculosis \\
\hline \multirow{4}{*}{ Liver diseases } & Acute fatty liver \\
\hline & Viral hepatitis \\
\hline & Intra hepatic biliary cirrhosis \\
\hline & Non-cirrhotic portal hypertension \\
\hline \multirow{3}{*}{ Renal diseases } & Urinary tract infection \\
\hline & Acute pyelonephritis \\
\hline & Acute renal failure \\
\hline \multirow{2}{*}{ Endocrinal disorders } & Gestational diabetes mellitus \\
\hline & Thyroid disorders \\
\hline \multirow{2}{*}{ Haematological disorders } & Anemia \\
\hline & Idiopathic thrombocytopenic purpura \\
\hline \multirow{2}{*}{ Protozoal infections } & Falciparum malaria \\
\hline & Vivax malaria \\
\hline \multirow{4}{*}{ Central nervous system } & Cerebrovascular accidents acute non-haemorrhagic infarct \\
\hline & Seizure disorder \\
\hline & Acute intraparenchymal bleed \\
\hline & Tuberculoma \\
\hline \multirow{2}{*}{ Other bacterial infections } & Acute gastroenteritis \\
\hline & Cholera \\
\hline \multirow{3}{*}{ Other viral infections } & Dengue fever \\
\hline & Chicken pox \\
\hline & Measles \\
\hline Autoimmune diseases & Systemic lupus erythematosus \\
\hline
\end{tabular}

Asymptomatic pregnant woman tested between 5 and 14 days of coming into direct contact of COVID-19 positive individual. Asymptomatic or detected to be COVID-19 positive during standard screening and referred to our institute for further management. Untraceable report of RT-PCR sample was sent to confirm the diagnosis. Single negative sample of RT-PCR was required to declare the patient as COVID-19 negative. Serology as a diagnostic procedure used only if RT-PCR was unavailable. Samples tested for other viruses, bacterial pneumonia, chlamydia and mycoplasma pneumoniae. Blood cultures taken to rule out secondary infection. ${ }^{9}$ Reinfection suspected as the patient developed flu like symptoms again and tested with RT-PCR to confirm the diagnosis.

\section{Statistical analysis}

Demographic variables that were continuous and normally distributed were expressed as means and standard deviations. Non-parametric continuous variables were expressed as medians with interquartile ranges (IQRs). All data were tested for normality with the appropriate result, presented as median versus mean. Categorical variables were expressed as numbers and percentages. In reporting 
outcomes, women are divided into 2 groups: those who were symptomatic and those who were asymptomatic and detected by screening.

\section{RESULTS}

In the present retrospective COHORT study, a total of 50 pregnant women included over a period of six months. The demographics of COHORT are presented in Table 2.

\section{Patient characteristics}

Maternal age ranged from 20 to 39 years with a mean age (SD) of 27.4 \pm 4.16 years with age range of 20-35 years. Mean period of gestation at the time of enrollment in the study was 33.26 \pm 8.35 . As per routine protocol/as per hospital policy, RT-PCR were conducted for all patients presented in OPD, a total of $33(66 \%)$ were found to be COVID-19 positive due to close contact. A total of 29 (58\%) women were found to be asymptomatic and 17 (34\%) had various other COVID-19 symptoms. On respiratory examination, a total of 41 women found to be asymptomatic/mild symptoms with $\mathrm{RR}<24 / \mathrm{m}$ and $\mathrm{SpO}_{2}$ $>94 \%$ in room air and 9 women symptomatic with mild to moderate pneumonia (Table 2).
Table 3 shows various COVID-19 symptoms observed in all the women at the time of enrolment in the present retrospective COHORT study. The most common symptom at presentation was sore throat $17(35 \%)$ followed by cough, myalgia and fatigue, that is, $13(26 \%)$ each. Fever at the time of admission was present in 9 $(18 \%)$ women and postpartum fever was observed in 4 $(8 \%)$ women. Less commonly reported symptoms included headache (4\%), abdominal pain (10\%) and chest tightness (8\%).

Various medical disorders observed during the study period is shown in Table 4. Most common problem found was PIH, that is, $26(52 \%)$ women suffering from hypertension followed by anemic women 15 (30\%).

Different laboratory findings of pregnant women were carried out during the study period which shows mean complete blood count was 10.47 \pm 1.26 (range 8.5-13), platelet count $1.90 \pm 0.39$ per microlitre (range 1.14-2.44), bilirubin $0.71 \pm 0.19 \mathrm{mg} / \mathrm{dl}$ (range 0.3-1), alkaline phosphatase $217.4 \pm 155.83$ IU/l (range 34-550), SGOT 57.16 \pm 51.50 units/l (range 12-190), SGPT 38.9 \pm 31.89 units/l (range 17-120) and random blood sugar was $114.76 \pm 38.48 \mathrm{mg} / \mathrm{dl}$ (range 72-212).

Table 2: Demographic and medical disorders distribution.

\begin{tabular}{|ll|}
\hline Parameters & Total no. $(\%)$ \\
\hline Mean age (in years) & $27.46 \pm 4.16$ (range 20-35) \\
\hline Mean period of gestation at diagnosis (in weeks) & $33.26 \pm 8.35$ (range 11-40.4) \\
\hline Close contact & $33(66)$ \\
\hline Health workers & 0 \\
\hline Comorbid illness & \\
\hline GDM & $17(34)$ \\
\hline Hypothyroid & $12(24)$ \\
\hline IHCP & $9(18)$ \\
\hline Preeclampsia & $9(18)$ \\
\hline Symptoms & \\
\hline Asymptomatic & $33(66)$ \\
\hline Symptomatic & $17(34)$ \\
\hline History of surgery & $4(8)$ \\
\hline Respiratory & \\
\hline Mild & $41(82)$ \\
\hline Moderate & $9(18)$ \\
\hline Severe & 0 \\
\hline
\end{tabular}

Table 3: Patients with symptoms upon presentation.

\begin{tabular}{|ll|}
\hline Parameters & Total no. $(\%)$ \\
\hline Fever at admission & $9(18)$ \\
\hline Fever postpartum & $4(8)$ \\
\hline Cough & $13(26)$ \\
\hline Sore throat & $17(34)$ \\
\hline Chest tightness & $4(8)$ \\
\hline Hemoptysis & 0 \\
\hline
\end{tabular}




\begin{tabular}{|ll|}
\hline Parameters & Total no. $(\%)$ \\
\hline Dyspnea & $8(16)$ \\
\hline Myalgia & $13(26)$ \\
\hline Headache & 2 \\
\hline Dizziness & 0 \\
\hline Fatigue & $13(26)$ \\
\hline Nausea & $4(8)$ \\
\hline Vomitting & 0 \\
\hline Abdominal pain & $5(10)$ \\
\hline Diarrhoea & 0 \\
\hline
\end{tabular}

Table 4: Medical disorders in pregnancy.

\begin{tabular}{|ll|}
\hline Parameters & Total no. $(\%)$ \\
\hline Pregnancy induced hypertension & $26(52)$ \\
\hline Gestational diabetes mellitus & $4(8)$ \\
\hline Epilepsy & $1(2)$ \\
\hline Hypothyroid & $3(6)$ \\
\hline Cardiac disease & $1(2)$ \\
\hline Anaemia & $15(30)$ \\
\hline Rh negative & $4(8)$ \\
\hline Bronchial asthma & $2(4)$ \\
\hline Depression & $6(12)$ \\
\hline Cancer & $2(4)$ \\
\hline Hepatitis & $5(10)$ \\
\hline Inflammatory bowel disease & $2(4)$ \\
\hline Sexually transmitted disease & $3(6)$ \\
\hline Renal problems & $6(12)$ \\
\hline
\end{tabular}

Table 5: Ultrasound examination of pregnant women.

\begin{tabular}{|ll|}
\hline Parameters & Mean \pm SD (range) \\
\hline Gestational age (in weeks) & $32.9 \pm 8.45$ \\
\hline BPD & $30.83 \pm 7.59$ \\
\hline AC & $32.17 \pm 3.30$ \\
\hline Fetal length & $34.21 \pm 2.17$ \\
\hline Fetal weight (in kgs) & $4.65 \pm 8.16$ \\
\hline Liquor & $11.03 \pm 4.39$ \\
\hline IUGR & $37(74 \%)$ \\
\hline
\end{tabular}

Table 6: Maternal outcome.

\begin{tabular}{|ll|}
\hline Parameters & Total no. $(\%)$ \\
\hline Hospital stay (in days) mean \pm SD (range) & $9.24 \pm 3.25$ \\
\hline Mode of delivery & \\
\hline LSCS & $29(58)$ \\
\hline Vaginal & $21(42)$ \\
\hline Indications of cesarean section & $10(20)$ \\
\hline Non reassuring fetal heart tones & $15(30)$ \\
\hline Repeat caesarean & $2(4)$ \\
\hline Arrest of labour & $2(4)$ \\
\hline Failure of induction & $4.48 \pm 2.24$ \\
\hline Postoperative hospital stay (in days) mean \pm SD (range) & $9(18)$ \\
\hline Postoperative complications & \\
\hline
\end{tabular}


Table 7: Perinatal outcome.

\begin{tabular}{|ll|}
\hline Parameters & Total no. $(\%)$ \\
\hline NICU stay & $8(16)$ \\
\hline Mortality & $2(4)$ \\
\hline
\end{tabular}

Ultrasound examination of the women showed that 37 $(74 \%)$ having intrauterine growth retardation (IUGR). Mean period of gestation was $32.9 \pm 8.45$ weeks. Mean BPD was $30.83 \pm 7.59$, AC $32.17 \pm 3.30$, fetal length $34.21 \pm 2.17$ and fetal weight was $4.65 \pm 8.16 \mathrm{kgs}$ (Table 5).

Mean hospital stay of these patients was $9.24 \pm 3.25$ days.

\section{Delivery characteristics}

A total of $29(58 \%)$ women underwent caesarean delivery. Caesarean deliveries were performed for non-reassuring fetal heart tones $(\mathrm{N}=10)$, repeat caesarean $(\mathrm{N}=15)$, arrest of labour ( $\mathrm{N}=2)$ and failure of induction $(\mathrm{N}=2) .21(42 \%)$ had normal vaginal delivery. Mean postoperative hospital stay was $4.48 \pm 2.24$ days. In 9 cases, postoperative complications were seen.

A total of 8 babies admitted to NICU due to various complications. Two babies expired during the study period.

\section{DISCUSSION}

In this retrospective COHORT study, we tried to find out the correlation of COVID-19 positive status in obstetric patients with medical disorders.

Various physiological changes which occurred during the period of pregnancy, make the mother more vulnerable to severe infections. ${ }^{10}$ Various anatomical changes like increase in the transverse diameter of the thoracic cage and an elevated level of the diaphragm, decrease maternal tolerance to hypoxia. ${ }^{11}$ Lung volume changes and vasodilation led to mucosal edema and increased secretions in the upper respiratory tract. Alterations in immune system increased the risk of infection in pregnant women to be infected by various viruses. ${ }^{12}$ It was recommended that pregnant women and their newborns should be assessed for various risk factors in the recent COVID-19 pandemic.

In the present retrospective COHORT study, mean age of the women was $27.46 \pm 4.16$ years. Mean period of gestation was $33.26 \pm 8.35$.

Similar to the present study, Breslin et al in their study reported 42 women with maternal age ranging from 20 to 39 years with a mean age $29.7 \pm 6.0$ years. ${ }^{14}$ Median gestational age in their study was 37 weeks.

In maximum number of women, $17(34 \%)$, sore throat was the commonest symptoms followed by cough, myalgia and fatigue, that is, $13(26 \%)$ each. Fever at the time of admission was present in $9(18 \%)$ women and postpartum fever was observed in $4(8 \%)$ women. A total of $33(66 \%)$ women were found to be asymptomatic and 17 (34\%) had various symptoms.

Chen et al conducted a similar study of small sample size in which they included only nine patients with caesarean section in 3rd trimester. ${ }^{15}$ They reported seven patients having fever, cough in four patients, myalgia in three patients, sore throat in two and malaise in two patients.

Knight et al prospective study was carried out by using the United Kingdom obstetric surveillance system (UKOSS) also found fever and cough as common symptoms in pregnant women with COVID-19 disease which was found to be similar to the present study. ${ }^{16}$

In our study, a total of $29(58 \%)$ women underwent caesarean delivery and $21(42 \%)$ had normal vaginal delivery. A total of 8 babies admitted to NICU due to various complications and two babies expired during the study period. No maternal mortality was seen in the present retrospective COHORT study.

Breslin et al in their study reported 18 women delivered out of which 4 were symptomatic at the time of initial presentation and 14 initially asymptomatic. ${ }^{14}$ Of these, 8 women (44.4\%) had a caesarean delivery and rest 10 women $(55.5 \%)$ had normal vaginal deliveries. These findings were found to be almost comparable to the present retrospective COHORT study.

Another study reported by Ayed et al showed 26.6\% of neonates preterm and $47.8 \%$ of neonates delivered through caesarean section. ${ }^{17}$ Similar to caesarean section reported in this study, our study reported $58 \%$ women with caesarean delivery.

Smith et al in their systematic review study reported low rates of maternal/neonatal mortality and ICU admissions in pregnant women with COVID-19. ${ }^{18}$ However, they further reported that COVID-19-positive pregnant women may be presented with fewer symptoms than the general population. The incidence of PTB, LBW, C-section, NICU admission appeared to be higher as compared to normal pregnant women.

In the present retrospective COHORT study, the most common medical disorder was pregnancy induced hypertension and its complication seen in $48 \%$ of the females, hypothyroidism in $22 \%$ and anaemia in $16 \%$ females. 
In $26(52 \%)$ women with pregnancy induced hypertension, perinatal morbidity was seen, $4(8 \%)$ intrauterine death and $12(24 \%)$ IUGR. Maximum number of perinatal morbidity was seen in anaemic women (56\% IUGR). Sore throat $(34 \%)$, cough $(26 \%)$, myalgia (26\%), fatigue $(26 \%)$, fever $(18 \%)$ and postpartum fever $(8 \%)$ were the most prevalent symptoms at the time of enrolment after diagnosis. These symptoms resolved within 4-6 weeks. After 4 weeks, $75 \%$ of participants had become asymptomatic and after 5 weeks, $15 \%$ more women were asymptomatic. After 6 weeks of treatment, symptoms persisted in 5\% of COVID-19 women and rest 5\% women no association was established with various symptoms. Average time from symptom onset to complete resolution of symptoms was 42 days.

Breslin et al in their study which was conducted for a 2week study period included 43 women. ${ }^{14}$ Out of these, 20 (69\%) reported COVID-19 symptoms as chief complaints and $9(31 \%)$ presented with primary obstetric complaints found to be symptomatic. The most common symptom in their study was dry cough $(\mathrm{N}=19,65.6 \%)$ followed by fever $(\mathrm{N}=14,48.3 \%)$ and myalgias $(\mathrm{N}=11,37.9 \%)$. Headache was found in only 8 women $(27.6 \%)$, shortness of breath was observed in 7 women $(24.1 \%)$ and chest pain in 5 women (17.2\%). In 29 women, 26 (89.7\%) had a combination of all these symptoms.

Due to lack of information about the coronavirus and increasing burden of the disease, it was important that information related to the disease may be shared to the general population in a proper manner. Data with regard to maternal and perinatal outcomes of pregnant women infected with the COVID-19 was found to be limited in the literature.

Limitation of the present retrospective COHORT study was small sample size with shorter duration. So, further studies with large sample size and duration are required to evaluate long-term maternal and perinatal outcomes in pregnant women.

\section{CONCLUSION}

Various types of medical disorders in obstetric patients are multifactorial and affects maternal and foetal outcome. If these symptoms detected at early stage, treatment protocol may be very easy and very few side effects may be observed to the mother and foetus. All these conditions suggests regular follow up, early detection of medical disorders, immediate action regarding treatment, regular follow up and to educate the women and aware them about these medical disorders and available treatment in time to improve maternal and foetal outcome.

In the present retrospective COHORT study, the majority of the pregnant women with COVID-19 infection had mild symptoms with no severe illness, but severe maternal and perinatal morbidity requires admission to intensive care unit.
Funding: No funding sources

Conflict of interest: None declared

Ethical approval: The study was approved by the Institutional Ethics Committee

\section{REFERENCES}

1. WHO. Fact sheet: Coronavirus disease (COVID-19) Pandemic, 2020. Available at: https://www.who.int/emergencies/diseases/novelcoronavirus-2019. Accessed on 10 June 2021.

2. Burrow G, Duffy T, Copel JA. Obstetric management of the high-risk patient. Medical complications during pregnancy. 6th ed. WB Saunders; 2004: 1-12.

3. Milind CA, Girish PB, Tushar TC, Abhijeet MC, Shashank TC, Hemant NC. A clinical study of medical disorders in pregnancy. Nat J Med Dent Res. 2016;4:257-63.

4. Shriastava S, Malik N. Medical disorders in pregnancy and pregnancy outcome a retrospective analysis. Int J Reprod Contracep Obstet Gynaec. 2018;7(6).

5. Clinical management. Available at: https://www.mohfw.gov.in/pdf/ClinicalManagement Protocol forCOVID19 dated 27062020.pdf.

6. ICMR-National Institute for Research in Reproductive Health. Fact sheet: Guidance for Management of Pregnant Women in COVID-19 Pandemic, 2020. Available at: http://www.nirrh.res.in/wpcontent/uploads/2020/04/Guidance-for-Managementof-Pregnant-Women-in-COVID-19-Pandemic.pdf. Accessed on 10 June 2021.

7. ICMR. Fact sheet: COVID-19 testing, 2020. Available at: documents/2020-0320covid19testv3.pdf

8. Good clinical practice recommendation on pregnancy with COVID-19 infection. FOGSI, 2020. Available at: https://www.fogsi.org/wpcontent/uploads/covid19/fogsi_gcpr_on_pregnancy_ with_COVID_19version1.pdf. Accessed on 10 June 2021.

9. Liang H, Acharya G. Novel corona virus disease (COVID-19) in pregnancy: what clinical recommendations to follow? Acta Obstet Gynecol Scand. 2020;99(4):439-42.

10. Goodnight WH, Soper DE. Pneumonia in pregnancy. Crit Care Med. 2005;33(10):390-7.

11. O'Day MP. Cardio-respiratory physiological adaptation of pregnancy. Semin Perinatol 1997;21(4):268-75.

12. Nelson-Piercy C. Respiratory disease. Handbook of Obstetric Medicine. Boca Raton: CRC Press; 2015: 371.

13. Chawla D, Chirla D, Dalwai S, Deorari AK, Ganatra A, Gandhi A, et al. Perinatal-neonatal management of COVID-19 infection-guidelines of the federation of obstetric and gynaecological societies of India (FOGSI), National neonatology forum of India 
(NNF), and Indian academy of pediatrics (IAP). Indian Pediatr. 2020;57(6):536-48.

14. Breslin N, Baptiste C, Gyamfi-Bannerman C, Miller R, Martinez R, Bernstein K, et al. Coronavirus disease 2019 among asymptomatic and symptomatic pregnant women: two weeks of confirmed presentations to an affiliated pair of New York City hospitals. Am J Obstet Gynecol MFM. 2020;2(2):100118.

15. Chen H, Guo J, Wang C, Luo F, Yu X, Zhang W, et al. Clinical characteristics and intrauterine vertical transmission potential of COVID-19 infection in nine pregnant women: a retrospective review of medical records. Lancet. 2020;395(10226):809-15.

16. Knight M, Bunch K, Vousden N, Morris E, Simpson $\mathrm{N}$, Gale $\mathrm{C}$, et al. Characteristics and outcomes of pregnant women admitted to hospital with confirmed SARS-CoV-2 infection in UK: national population based COHORT study. Br Med J. 2020;369:2107.
17. Ayed A, Embaireeg A, Benawadh A, Al-Fouzan W, Hammoud M, Al-Hathal M, et al. Maternal and perinatal characteristics and outcomes of pregnancies complicated with COVID-19 in Kuwait. BMC Pregnan Childbirth. 2020;20:754.

18. Smith V, Seo D, Warty R, Payne O, Salih M, Chin $\mathrm{KL}$, et al. Maternal and neonatal outcomes associated with COVID-19 infection: a systematic review. PLoS One. 2020;15(6):0234187.

Cite this article as: Goyal G, Tongaria K, Kumar S, Thakran BK, Yadav M. Maternal and perinatal morbidity and mortality in COVID-19 positive obstetric patients with medical disorders in tertiary care hospital in North India. Int J Reprod Contracept Obstet Gynecol 2021;10:3478-85. 